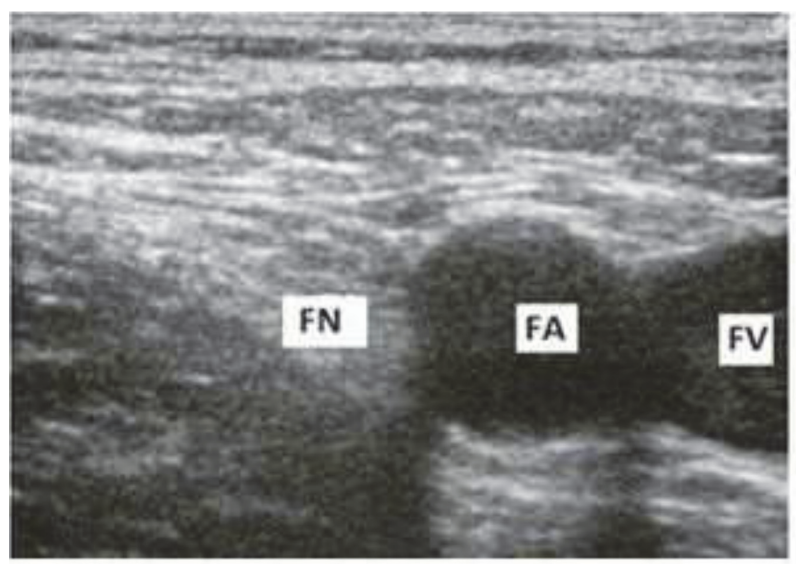

Abstract 172 Figure 3

- Conclusions Combined Sciatic and Femoral Nerve block is an effective and viable alternative to either General, Spinal or Epidural Anaesthesia as it provides better hemodynamic stability and good post operative analgesia in high risk patients without resulting in any adverse incidents.

\section{PERIPHERAL NERVE BLOCKS ALLOW HIP FRACTURE SURGICAL MANAGEMENT IN AN EXTREMELY FRAGILE PATIENT: NO PENG NO GAME}

F Gargano, A Strumia*, F Costa, G Pascarella, S Rizzo, V Antinolfi, G Biondo, G Rizzello, FE Agrò. Università Campus Biomedico di Roma, Rome, Italy

\subsection{6/rapm-2021-ESRA.173}

Background and Aims Hip fracture represents a frequent cause of disability in the elderly [1]. Early surgery $(<48 \mathrm{~h})$ has been proven to be associated with lower risk of mortality and morbidity [2]. Regional anaesthesia seems to be the best option, but there is no consensus when a central block is not indicated [3]. We present a case of a multimorbidity patient under anticoagulant drug, underwent hip fracture surgical repair with peripheral nerve blocks.

Methods Female 84 y.o. Atrial fibrillation, PMK wearer, hypertension, renal failure, CODP, cerebrovascular disease, OSAS, confusion and psychomotor agitation.

Echocardiogram: severe systolic function reduction, estimated election fraction $23 \%$.

Right sub-capital hip fracture, candidates for hip endoprosthesis.

Anticoagulant therapy with apixaban with no data on last assumption; thromboelastogram showed augmented values, so a central block was not indicated. Patient's multimorbidity highly elevated the risk of general anaesthesia.

With patient consent, peripheral blocks and sedation were considered. Ultrasound guided Pericapsular Nerve Group Block (PENG) was performed (ropivacaine 0,5\% $20 \mathrm{ml}+$ dexamethasone $4 \mathrm{mg}$ ), to block femoral, obturator and accessory obturator nerves, which innervate the hip [4]. Lateral femoral cutaneous nerve was also blocked, to cover surgical incision. Moderate sedation was given, maintaining spontaneous breath. Results Surgical procedure was carried out uneventfully and without pain, parameters were good and stable during and after surgery.

Conclusions Regional anaesthesia could be the key in fragile patients. Knowledge of anatomy and different techniques is

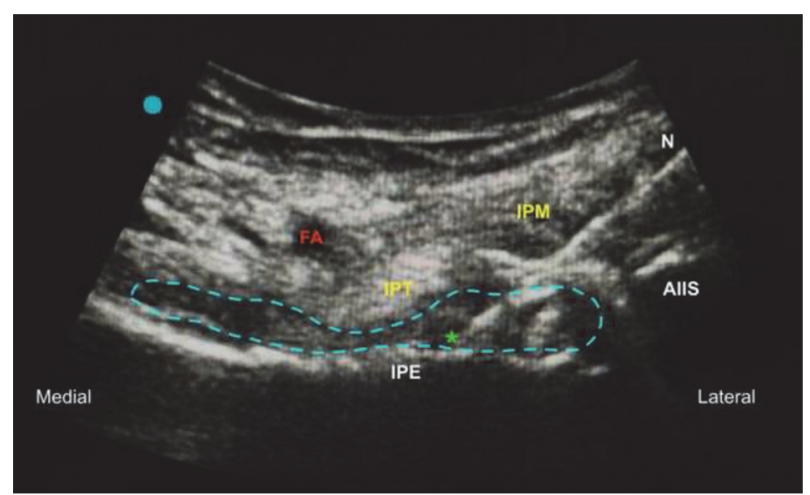

Abstract 173 Figure 1

$\mathrm{FA}=$ femoral artery; IPM=iliopsoas muscle; IPT=ilipsoas tendon; $\mathrm{N}=$ needle; IPE=iliopubic eminence; AllS=anterior inferior iliac spine.

fundamental to tailor the anaesthetic plan on patient conditions and surgical approaches.

\section{PERIOPERATIVE JOURNEY OF PATIENTS HAVING UPPER LIMB NERVE BLOCKS- A SERVICE EVALUATION AND QI PROJECT}

${ }^{1} \mathrm{M}$ Singh* ${ }^{2}{ }^{2} \mathrm{~S}$ Dondapati, ${ }^{2} \mathrm{~A}$ Singh. ${ }^{1}$ Stoke School of Anaesthesia, Stoke-on-Trent, UK; ${ }^{2}$ New Cross Hospital, Wolverhampton, UK

\subsection{6/rapm-2021-ESRA. 174}

Background and Aims Regional anaesthesia (RA) improves postoperative analgesia and can contribute to a reduction in postoperative nausea and vomiting (PONV). RA can improve value of care by reducing length of stay and enhancing patient satisfaction $^{1}$.

Regional nerve blocks are commonly performed for upper limb day case surgeries at our centre. We did a prospective patient survey on perioperative experience of upper limb surgery with nerve blocks.

Methods Patients who had regional nerve blocks for upper limb surgery were given proforma to fill after surgery with pre-stamped envelopes. We included 28 proformas returned from March to June 2019 for the survey.

Results All patients received anaesthetic information pre-surgery which included pre-assessment nurse (7/28), information leaflet $(2 / 28)$ and both (19/28). One patient found information provided unsatisfactory. Quarter of (8/28) patients reported severe pain and PONV during first 24 hours and timings of first oral pain relief was variable with non-standardised analgesics on prescription.

Most of patients (92\%) would prefer to have nerve block again.

Conclusions Results showed that post op pain prescription varied. Although most patients were satisfied with nerve block information but could be improved. We recommended to standardise post-operative discharge prescription. We suggested to produce patient video about the perioperative journey of nerve blocks for upper limb surgery which has been produced and will be implemented soon as delayed by pandemic.

Regular patient satisfaction surveys are needed for service evaluation and improvement. 